\title{
Investigation of an Organomagnesium Based [3 + 3] Annelation to Pyrans and its Application in the Synthesis of Rhopaloic Acid A
}

\author{
Julien C. R. Brioche, Katharine M. Goodenough, David J. Whatrup \\ and Joseph P. A. Harrity*
}

\section{Supporting Information}

\section{Table of Contents}

${ }^{1} \mathrm{H} /{ }^{13} \mathrm{C}$ NMR Spectrum of $\mathbf{1 3}$

${ }^{1} \mathrm{H} /{ }^{13} \mathrm{C}$ NMR Spectrum of $\mathbf{1 5 a}$

${ }^{1} \mathrm{H} /{ }^{13} \mathrm{C}$ NMR Spectrum of $\mathbf{1 5 b}$

${ }^{1} \mathrm{H} /{ }^{13} \mathrm{C}$ NMR Spectrum of $\mathbf{1 9}$

${ }^{1} \mathrm{H} /{ }^{13} \mathrm{C}$ NMR Spectrum of $\mathbf{2 0}$

6

${ }^{1} \mathrm{H} /{ }^{13} \mathrm{C}$ NMR Spectrum of $\mathbf{2 1}$

${ }^{1} \mathrm{H} /{ }^{13} \mathrm{C}$ NMR Spectrum of $\mathbf{2 2}$

${ }^{1} \mathrm{H} /{ }^{13} \mathrm{C}$ NMR Spectrum of $\mathbf{2 3}$

8

${ }^{1} \mathrm{H} /{ }^{13} \mathrm{C}$ NMR Spectrum of $\mathbf{2 4}$

${ }^{1} \mathrm{H} /{ }^{13} \mathrm{C}$ NMR Spectrum of $\mathbf{2 5}$

${ }^{1} \mathrm{H} /{ }^{13} \mathrm{C}$ NMR Spectrum of 7-(tert-butyldiphenylsilyloxy)-

2-methyleneheptane-1,5-diol

${ }^{1} \mathrm{H} /{ }^{13} \mathrm{C}$ NMR Spectrum of trans/cis 28

${ }^{1} \mathrm{H} /{ }^{13} \mathrm{C}$ NMR Spectrum of $\mathbf{3 2}$

${ }^{1} \mathrm{H} /{ }^{13} \mathrm{C}$ NMR Spectrum of 33

Crude ${ }^{1} \mathrm{H}$ NMR Spectrum of intermediate aldehyde

${ }^{1} \mathrm{H} /{ }^{13} \mathrm{C}$ NMR Spectrum of $\mathbf{3 4}$

17

${ }^{1} \mathrm{H} /{ }^{13} \mathrm{C}$ NMR Spectrum of $\mathbf{3 5}$

Comparative data for rhopaloic acid A 35 
Brioche et al Page 2
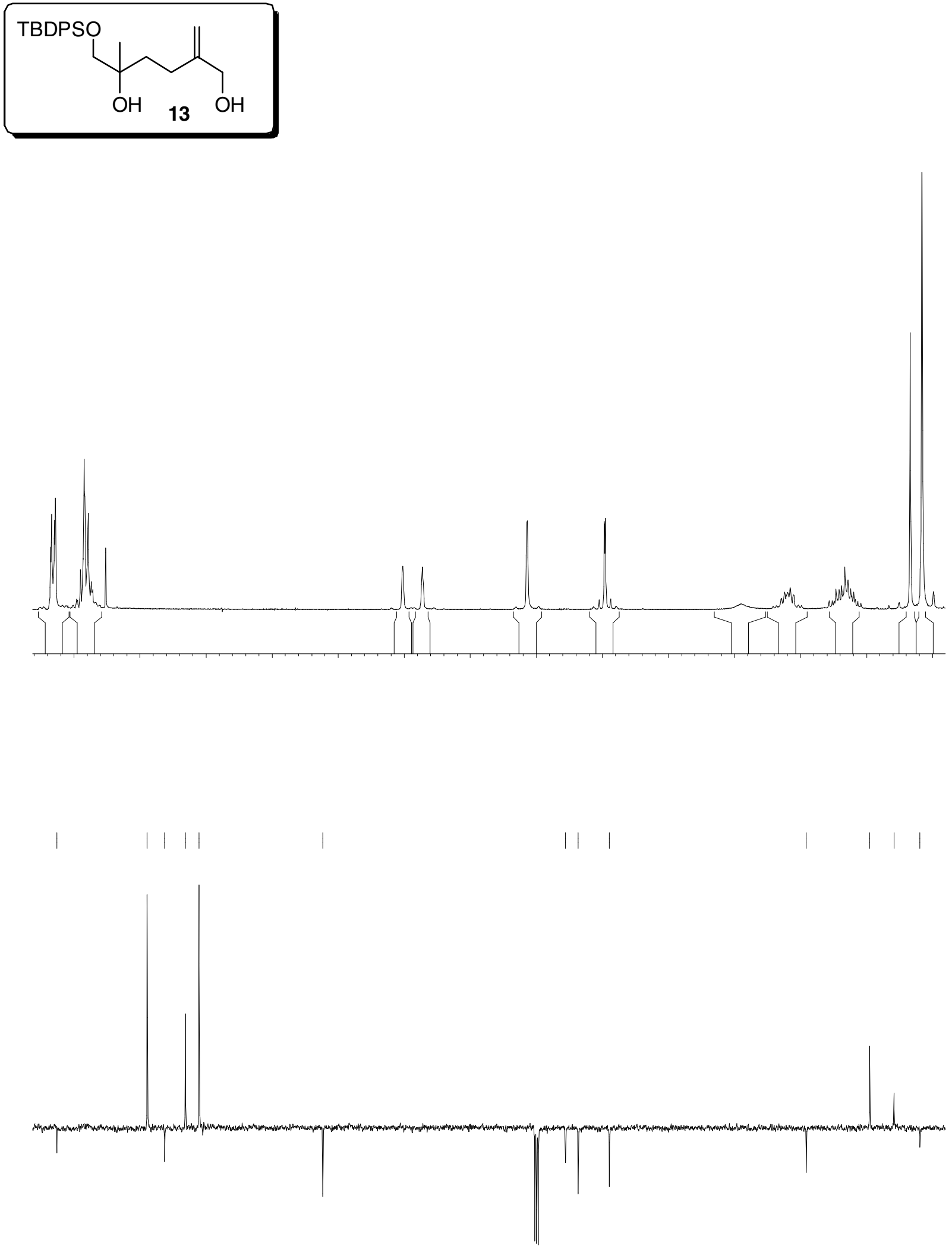
Brioche et al Page 3
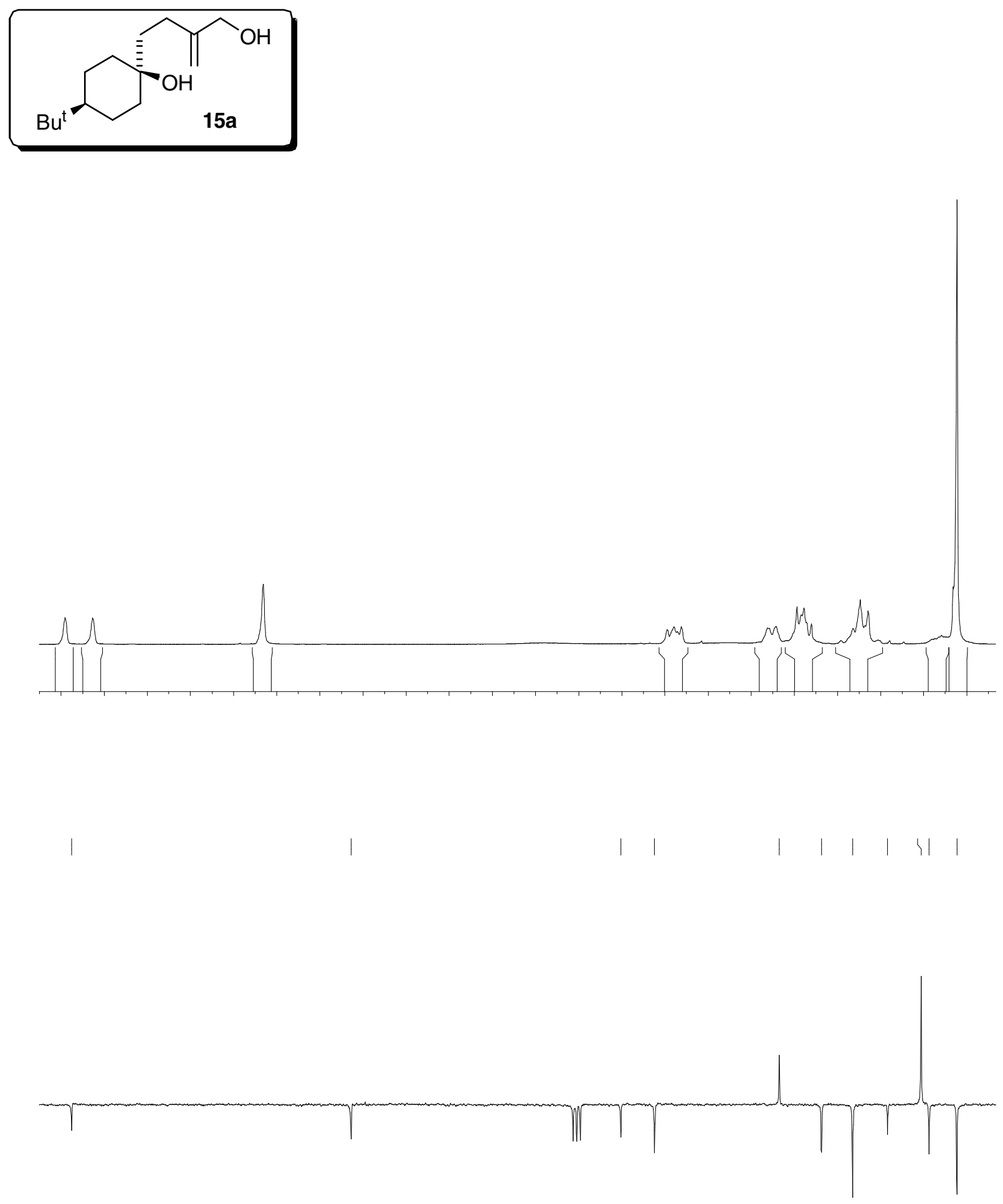
Brioche et al Page 4
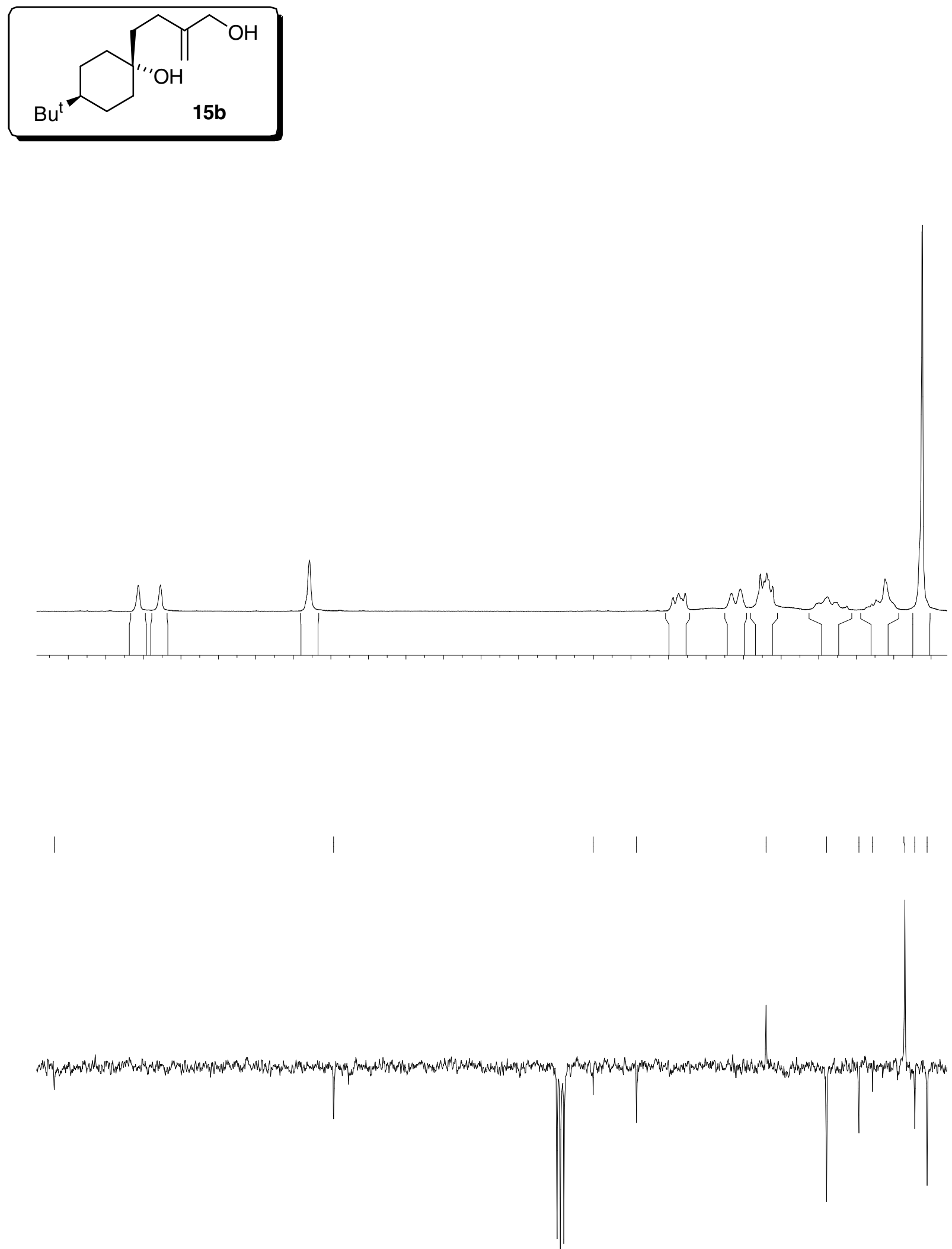
Brioche et al Page 5
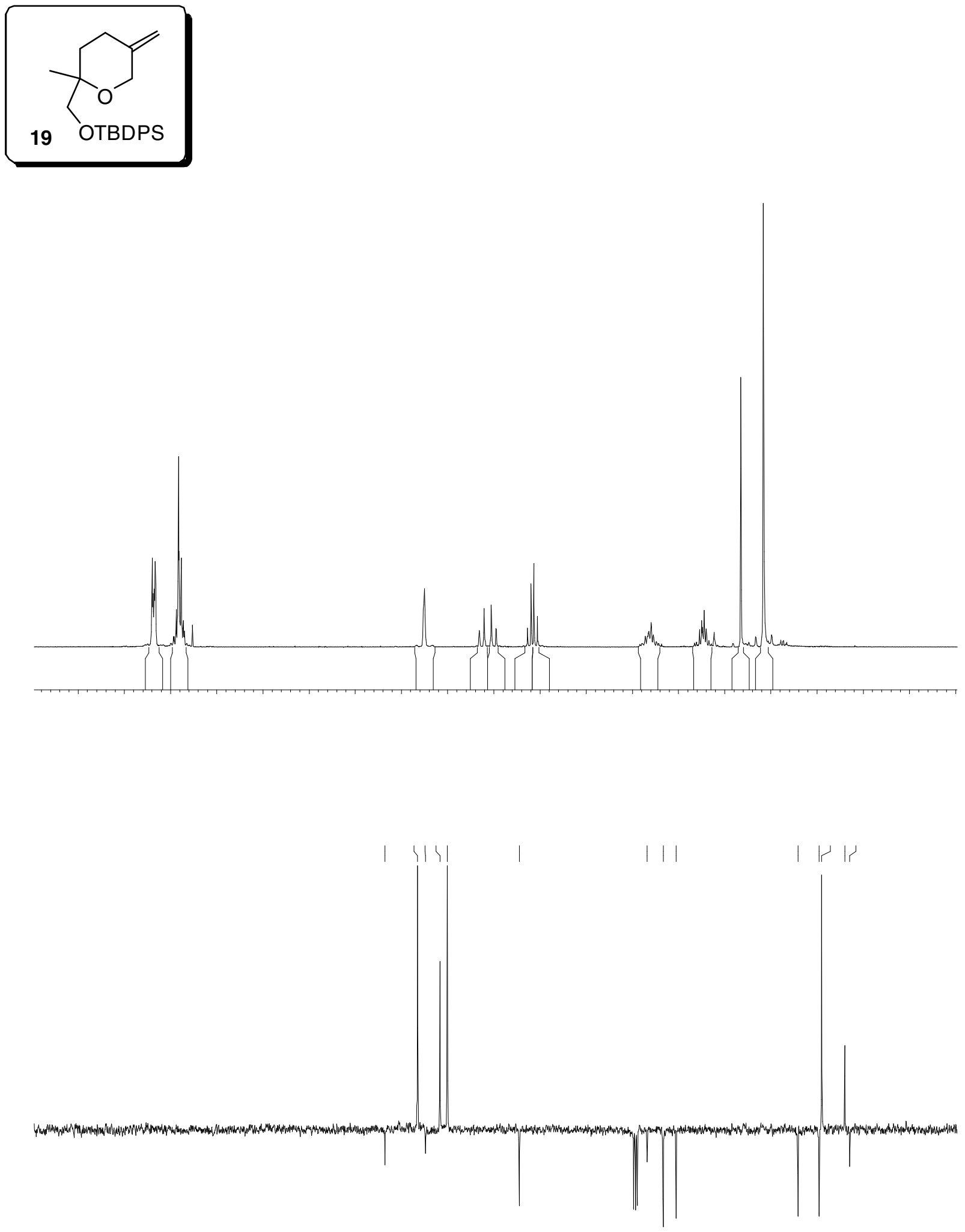
Brioche et al Page 6
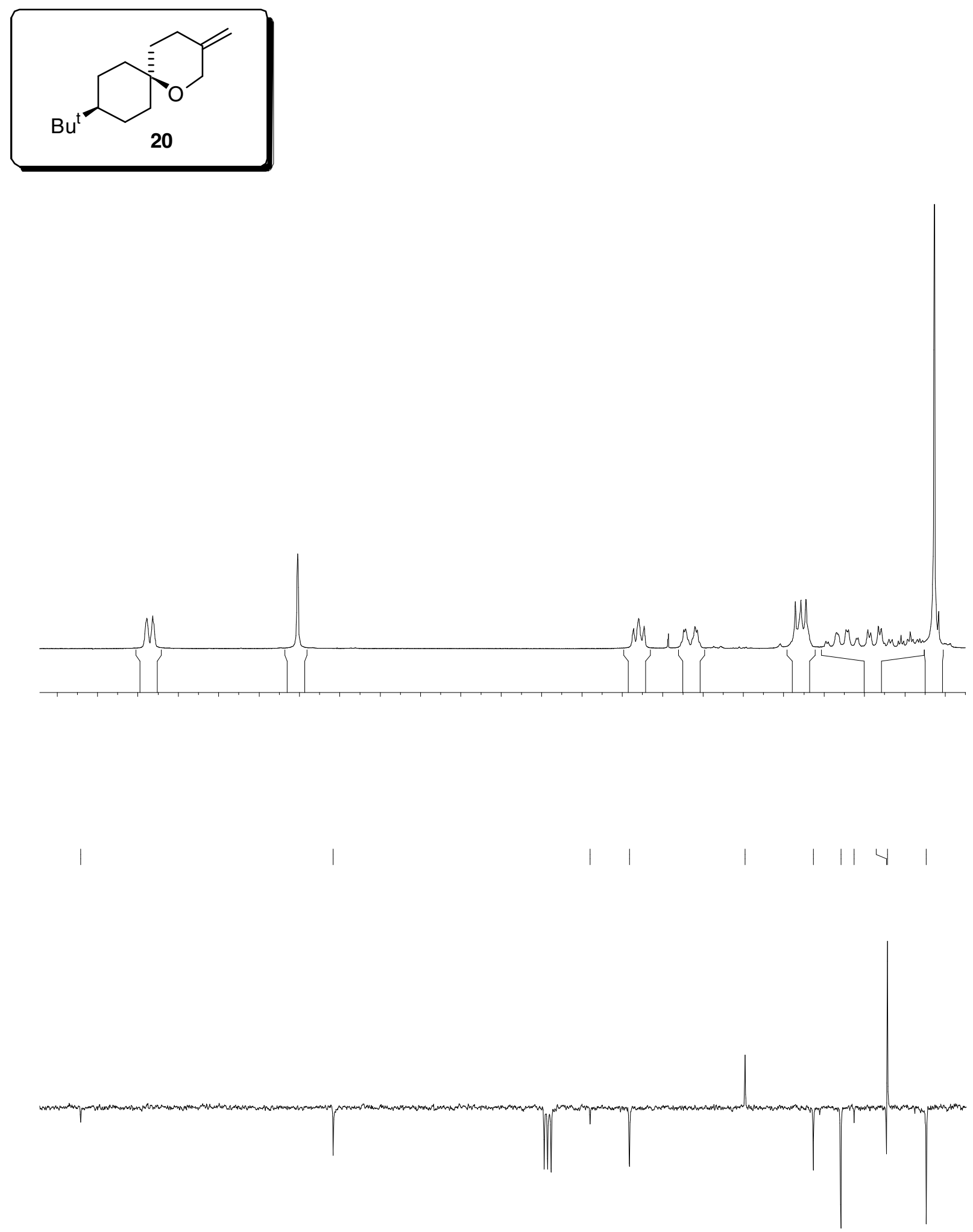
Brioche et al Page 7
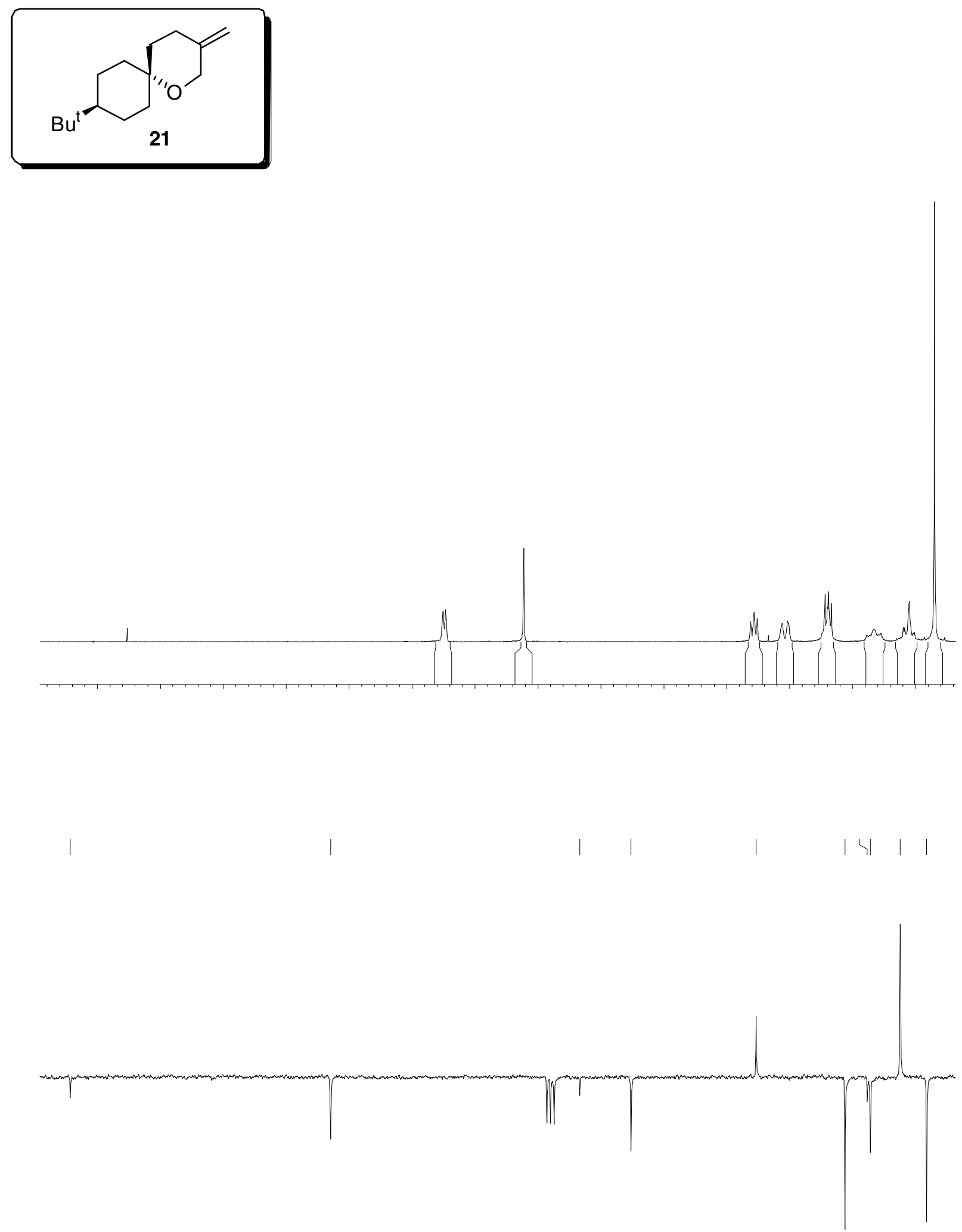
Brioche et al Page 8
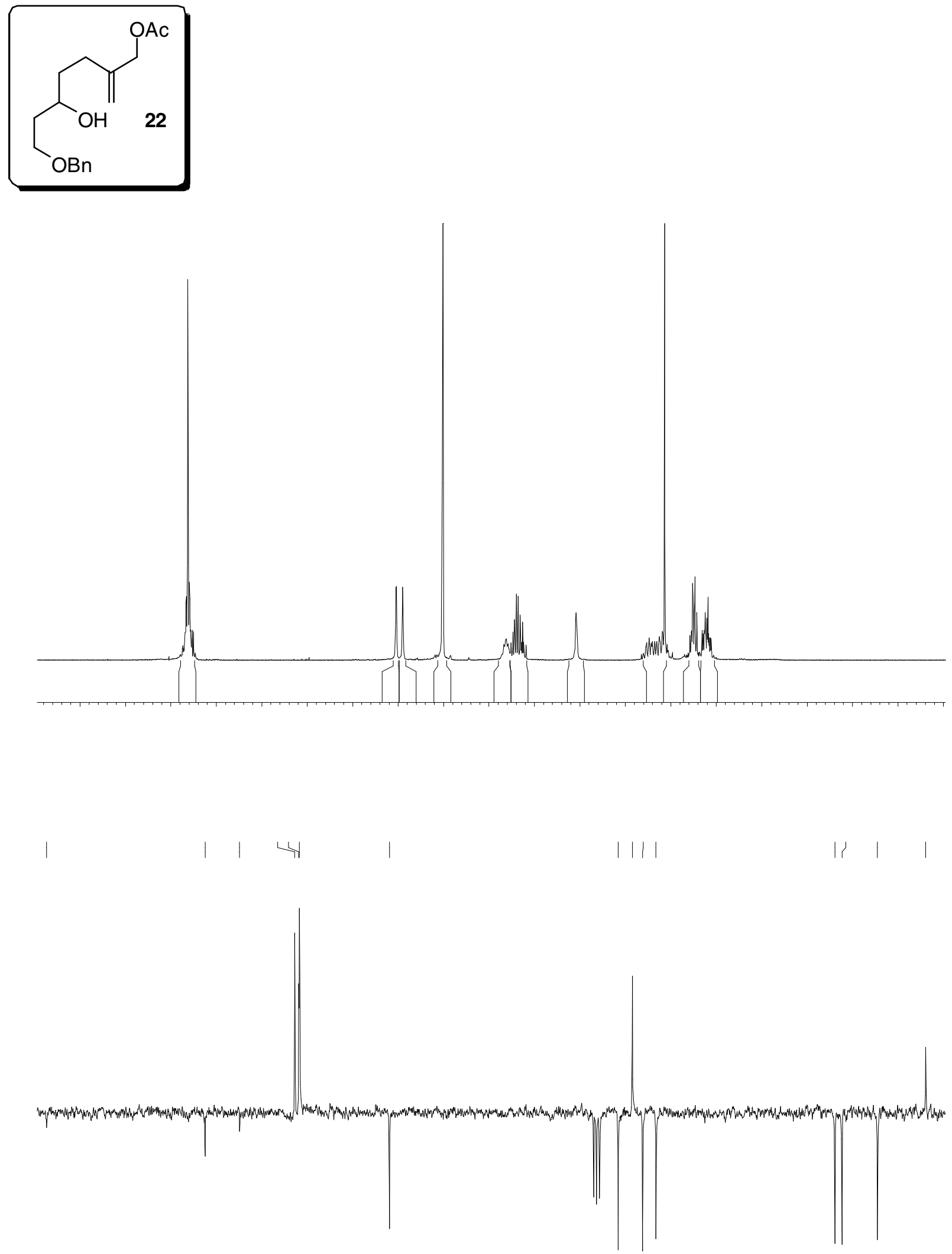

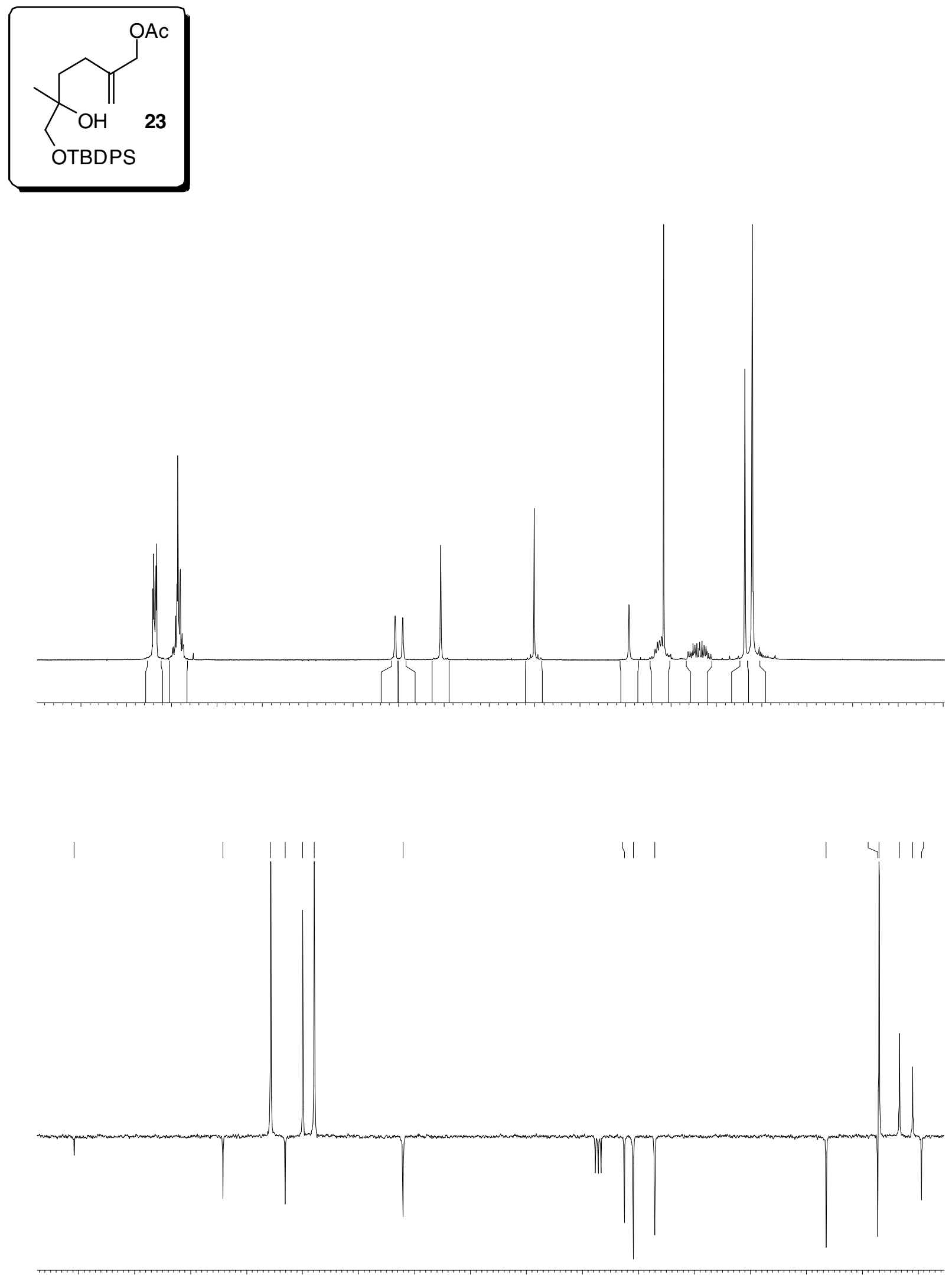

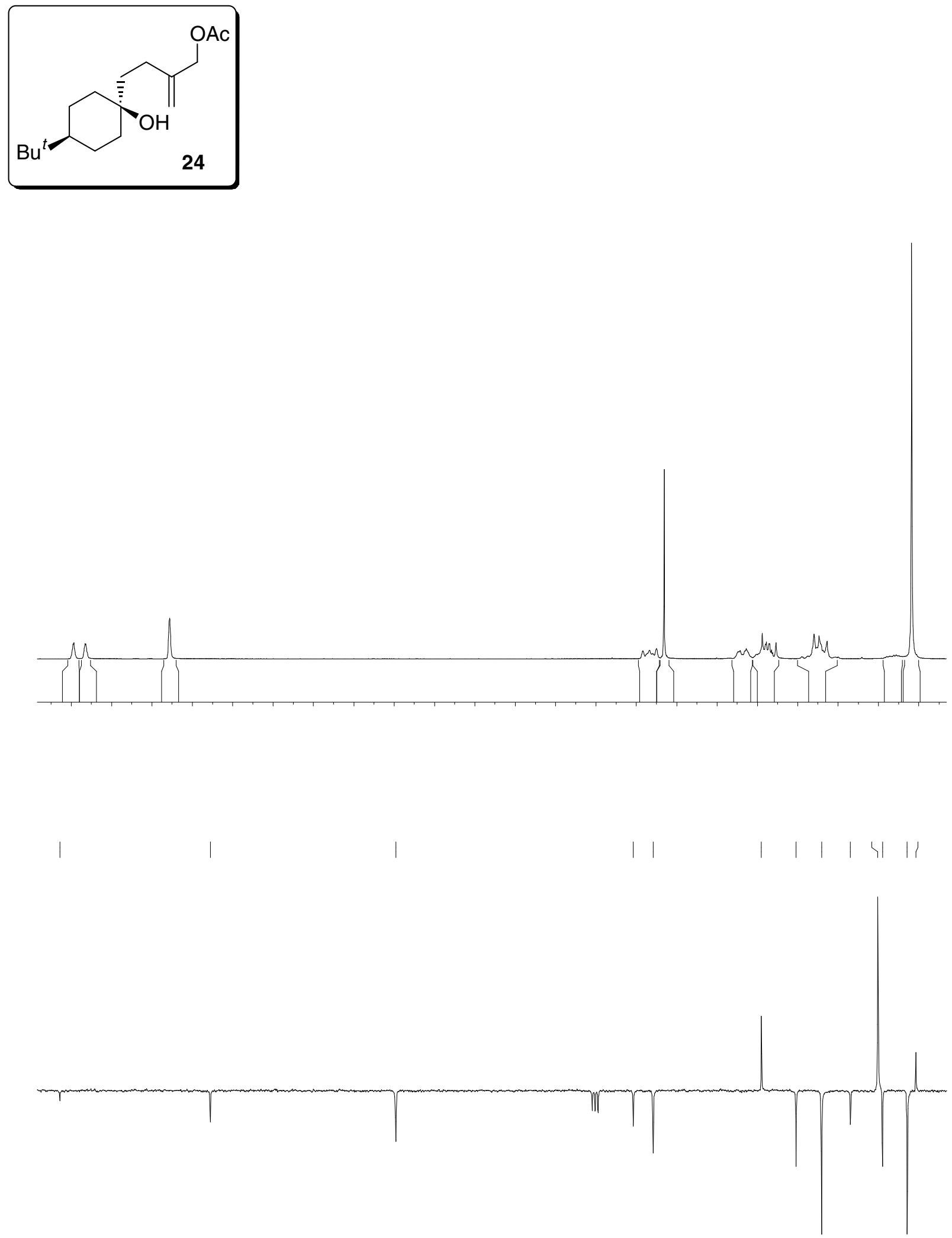

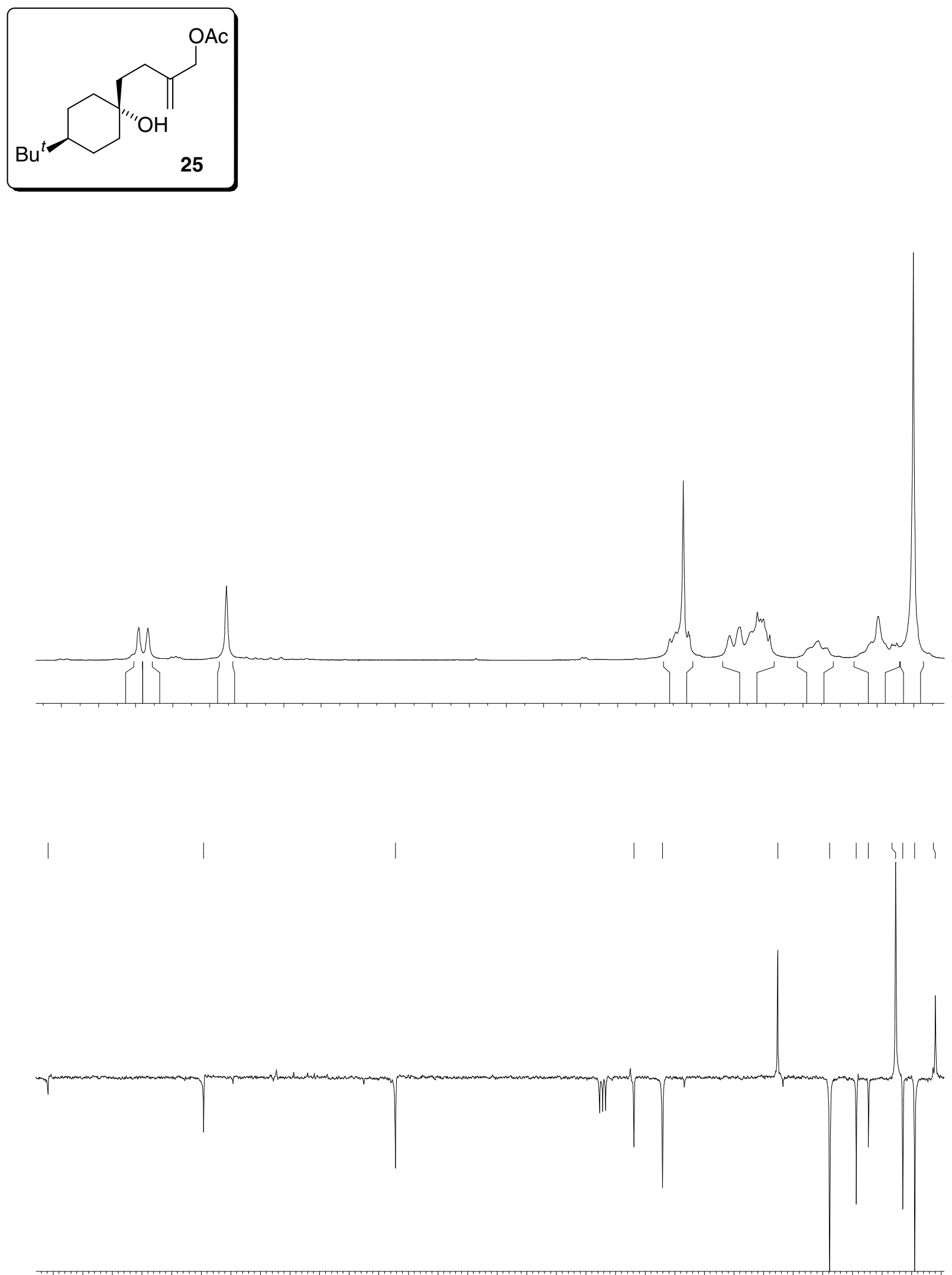

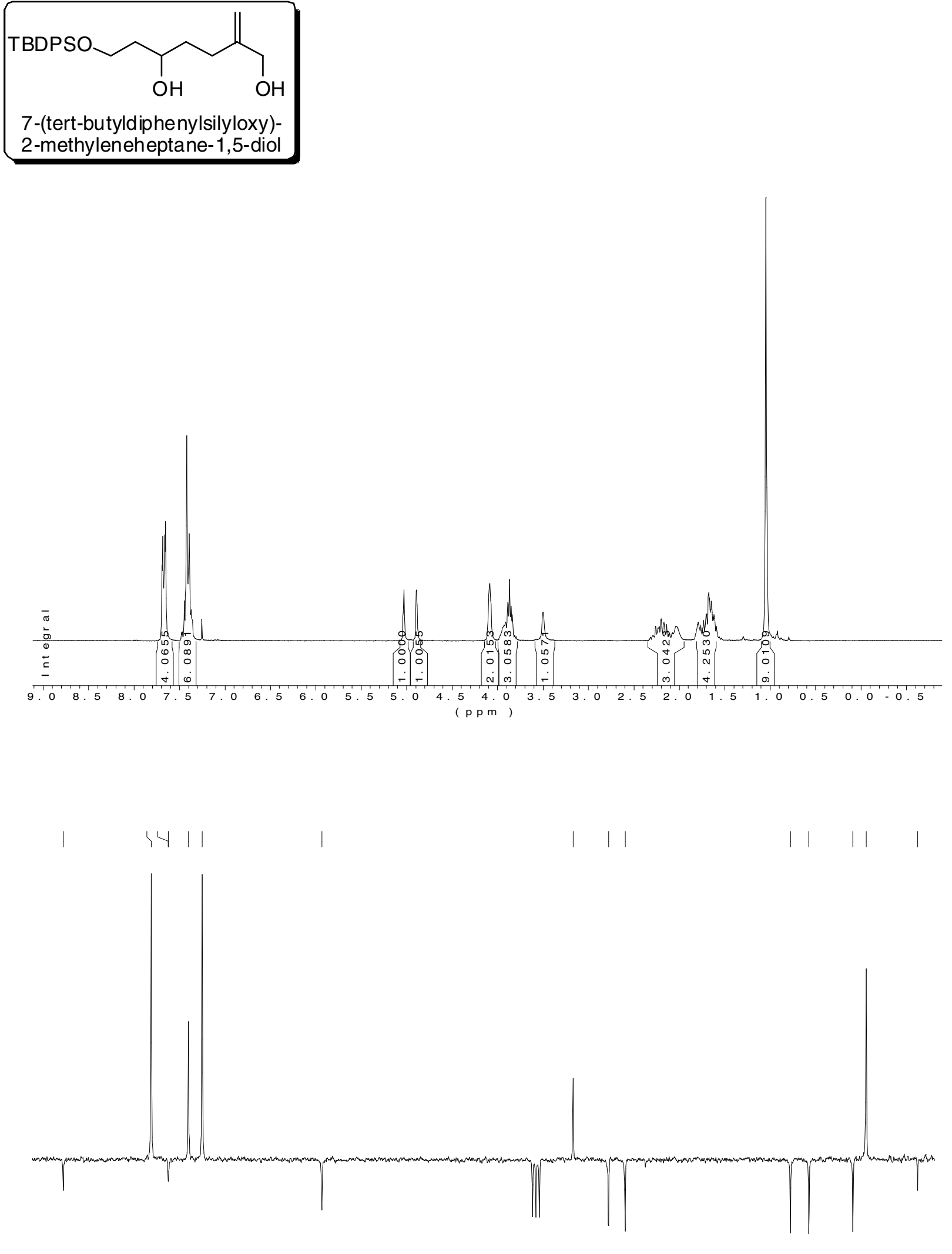

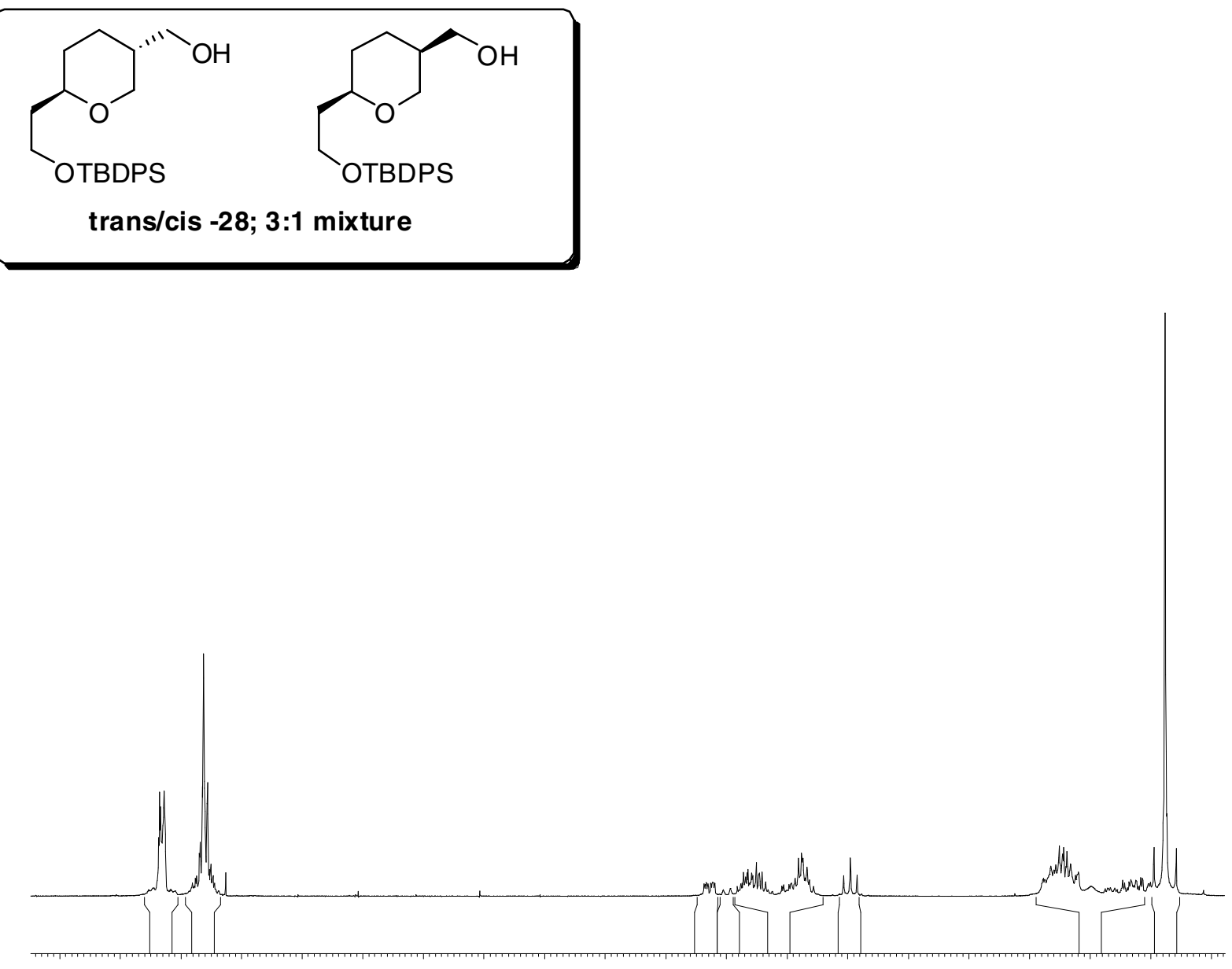

411

$\checkmark|1| H$

He

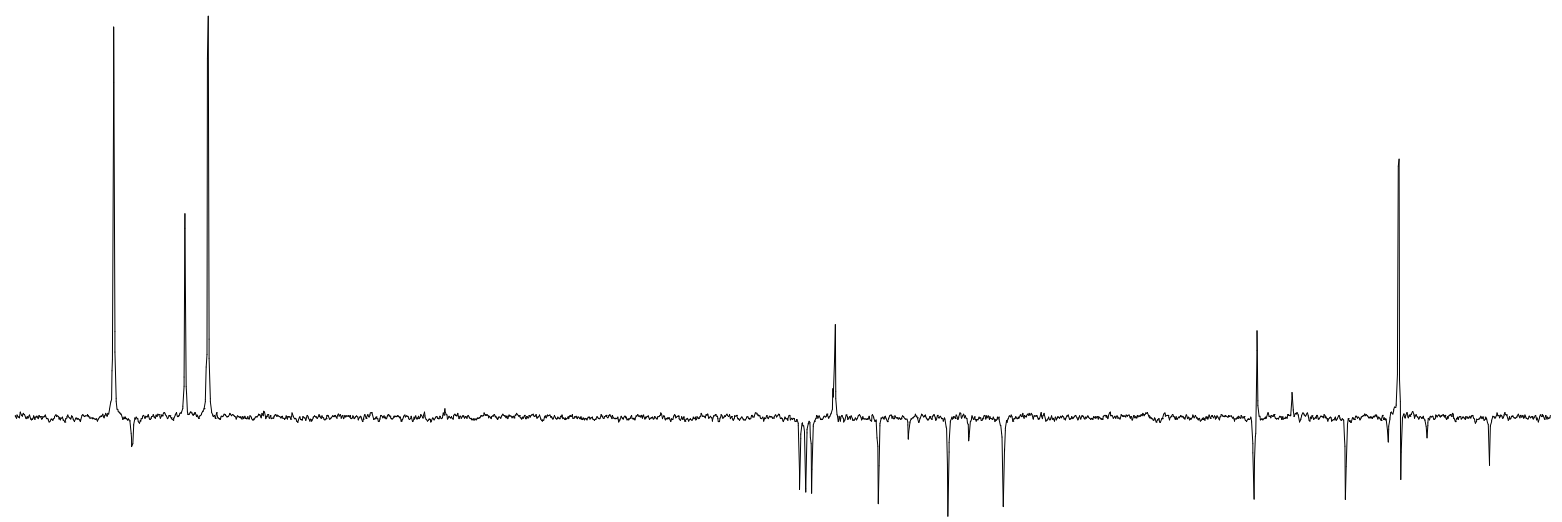


Brioche et al Page 14
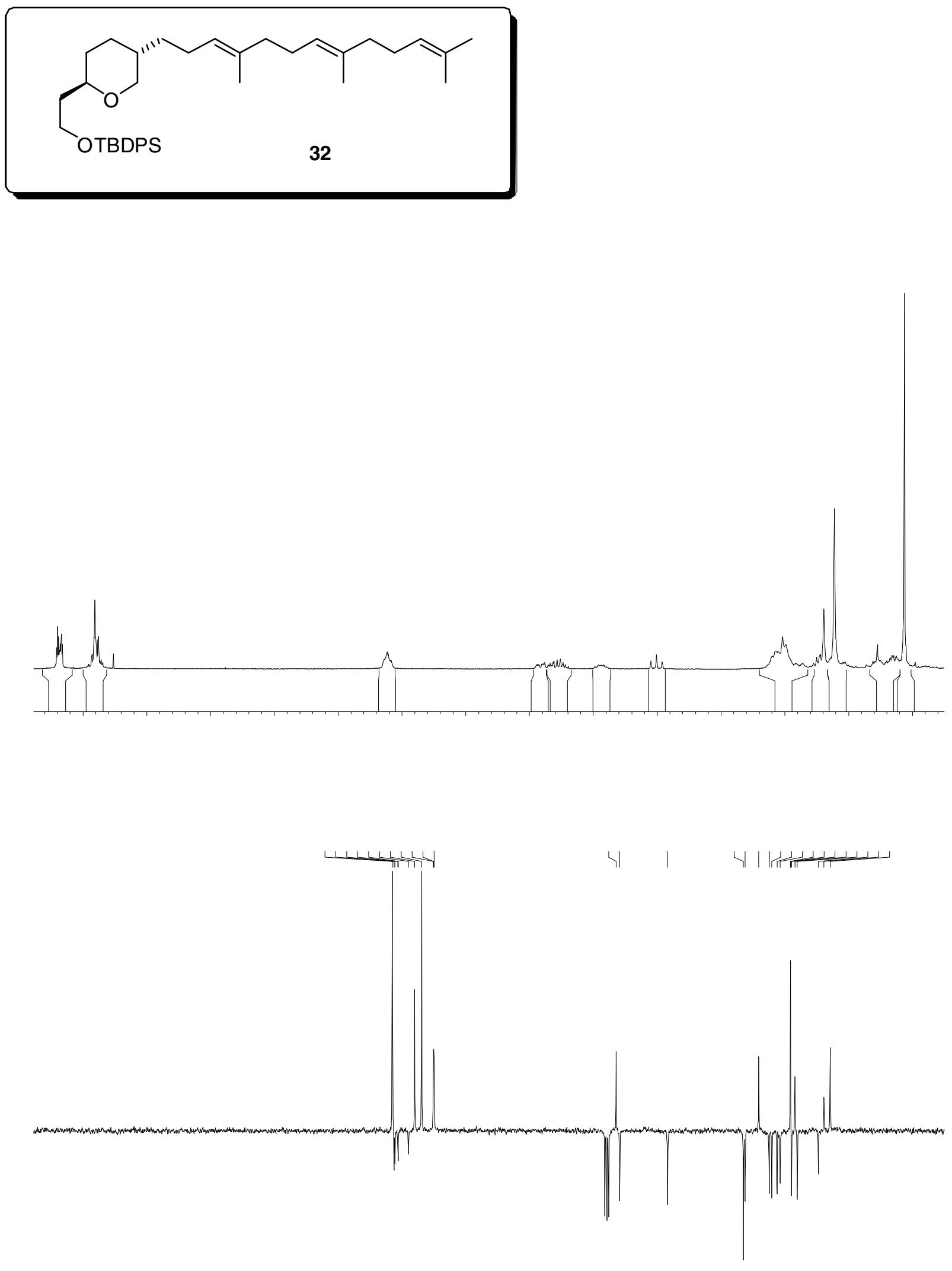
Brioche et al Page 15
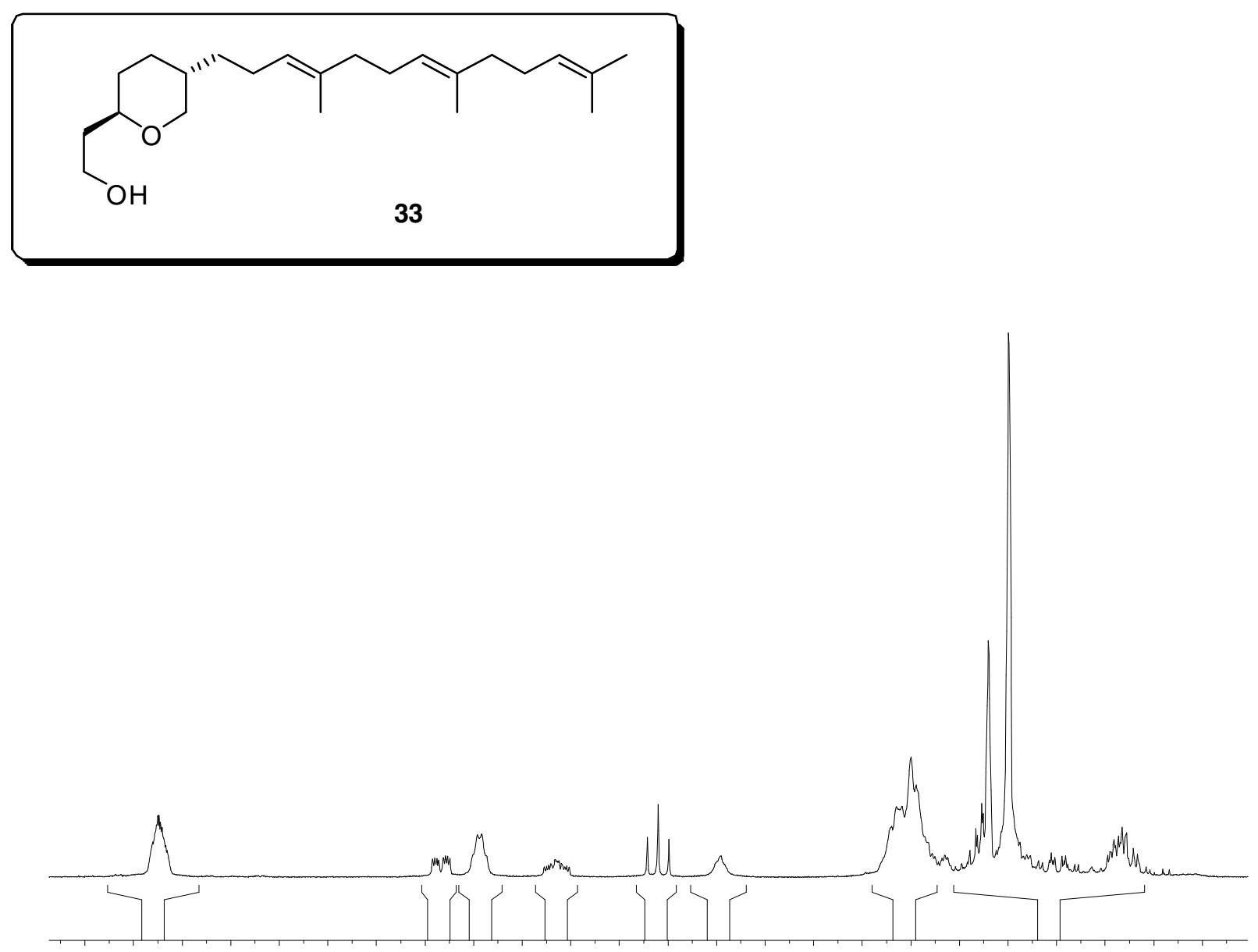

414

| |

||

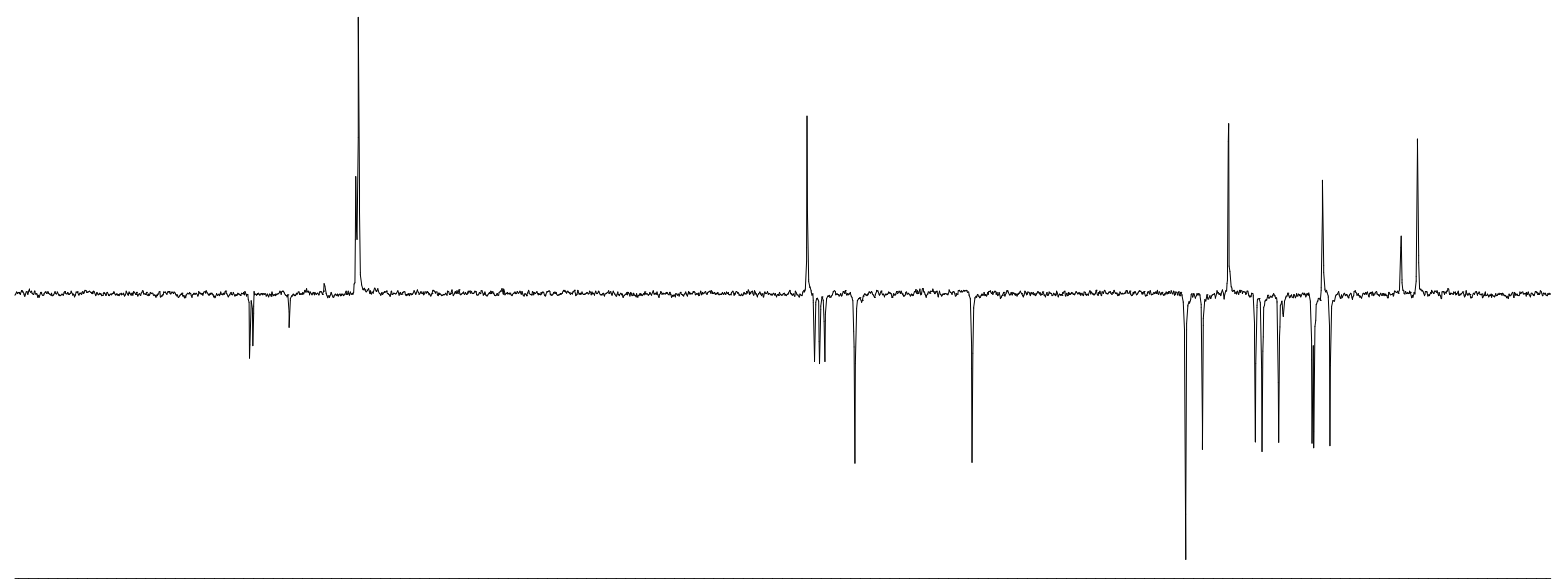



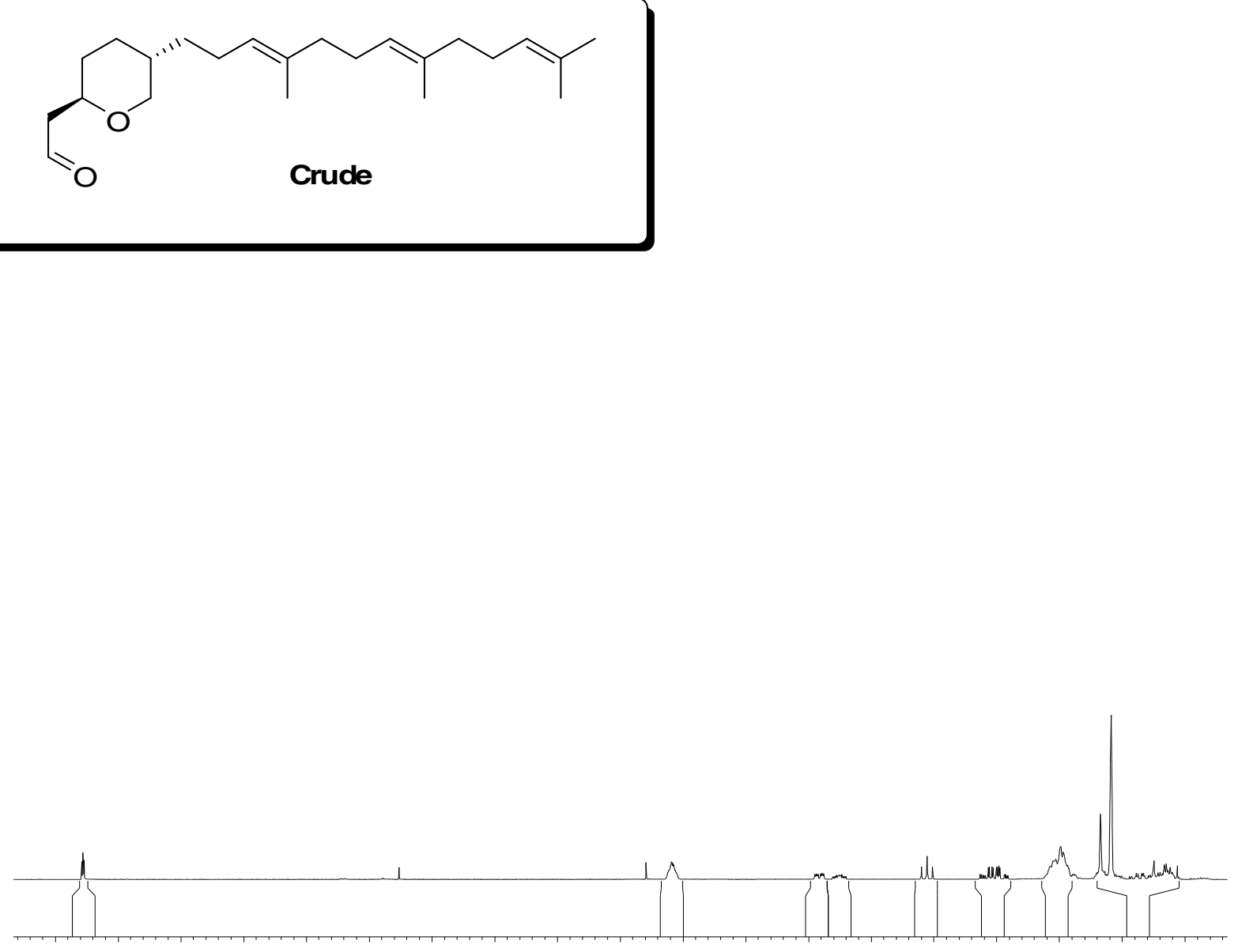
Brioche et al Page 17
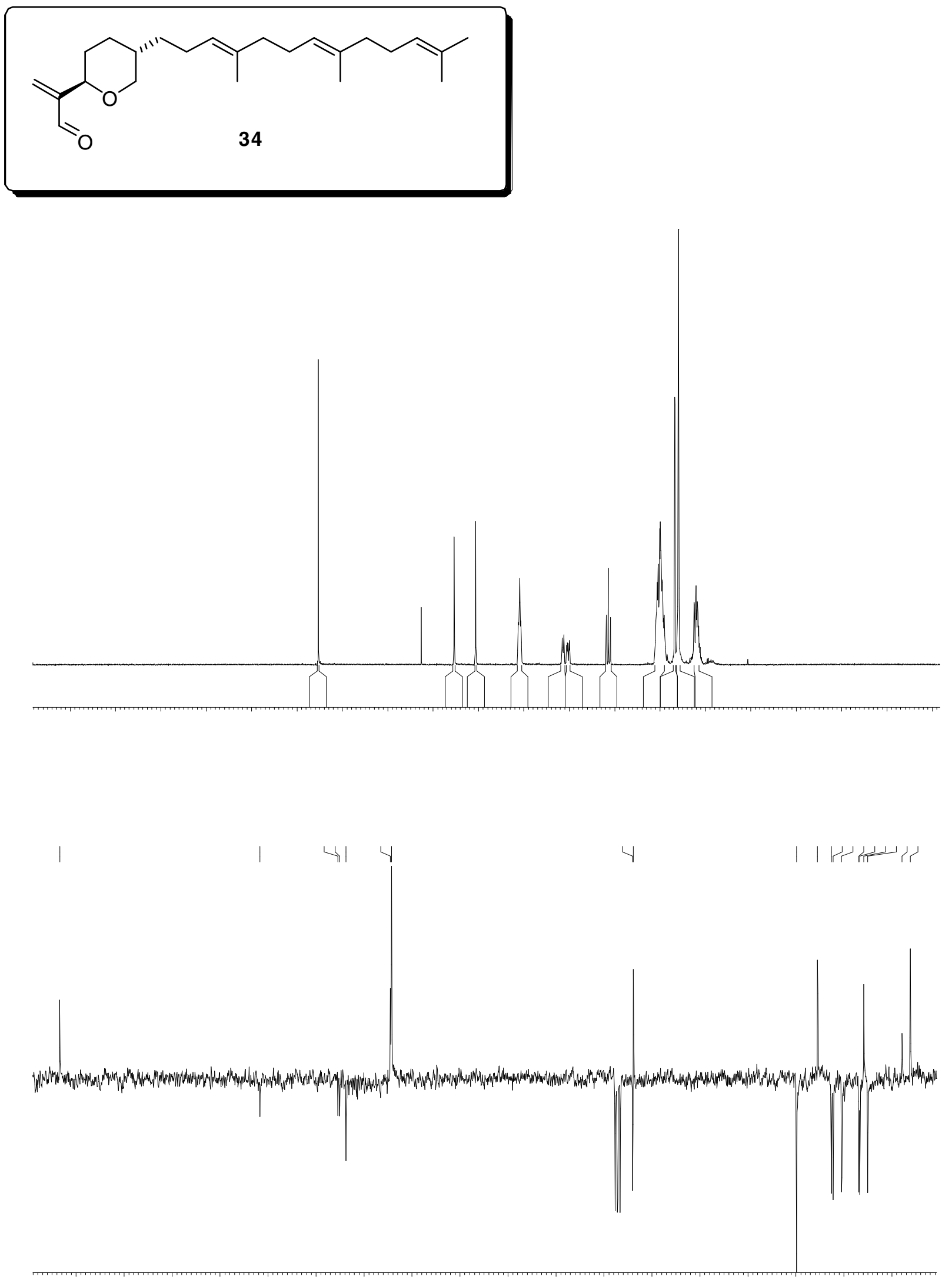

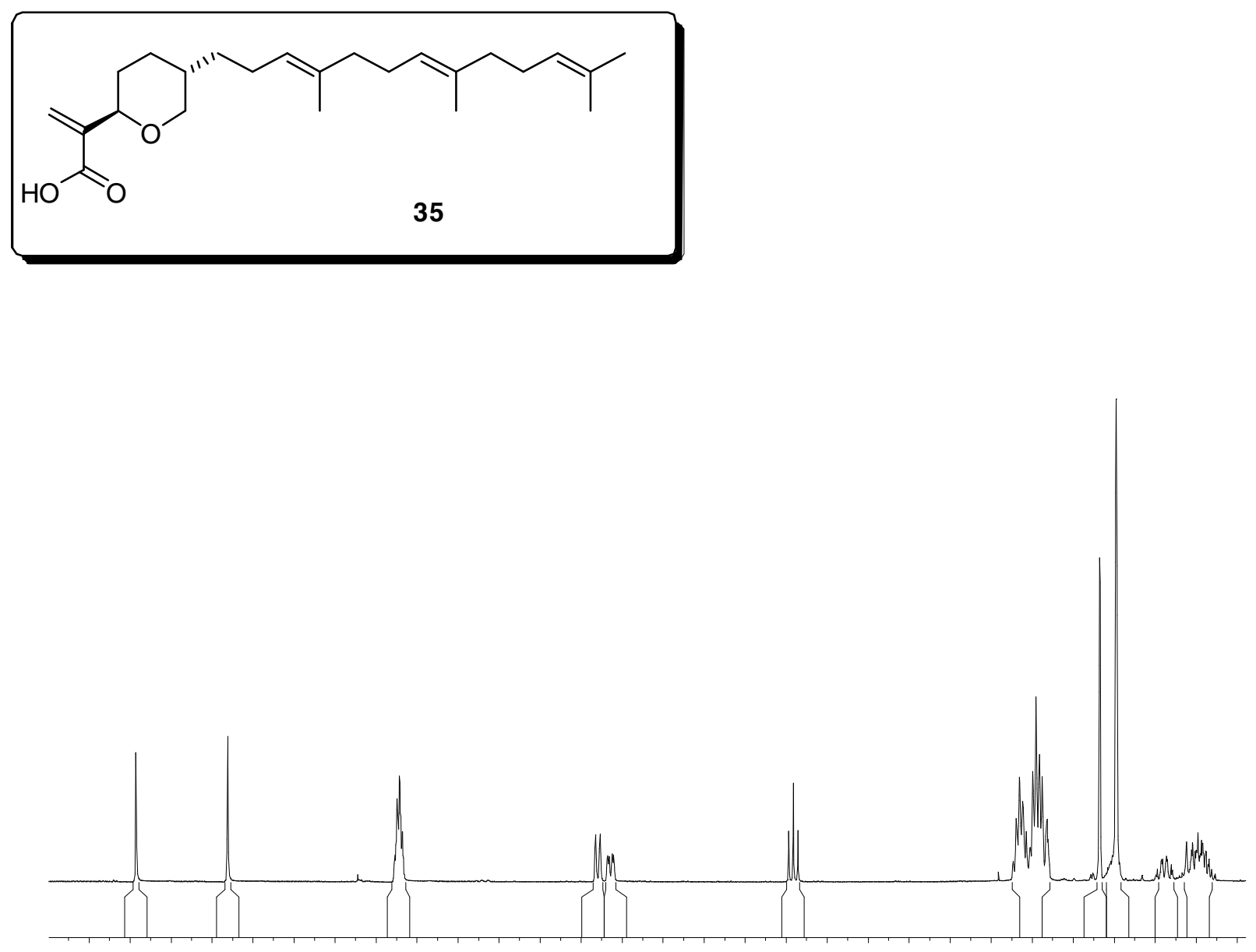

$1 \quad 1434$

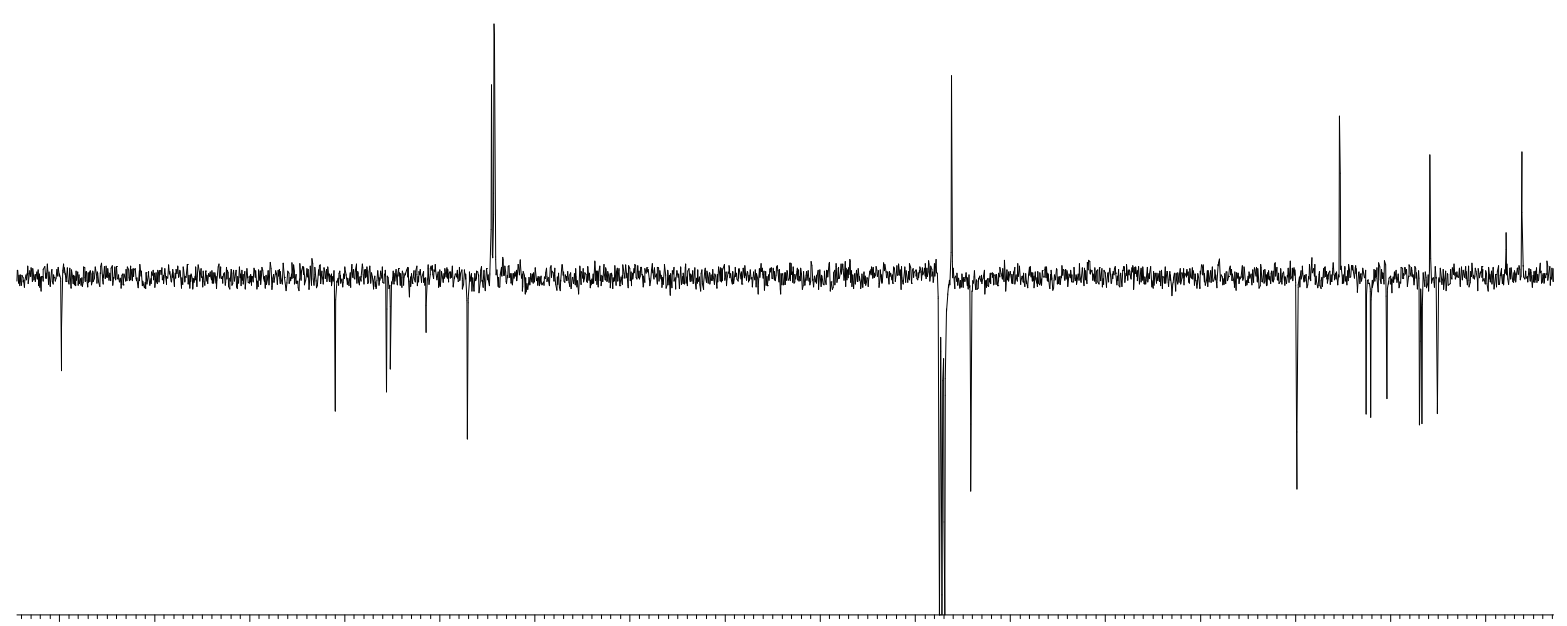




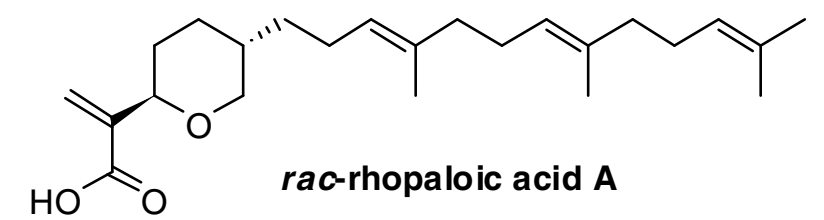

\begin{tabular}{|c|c|c|c|}
\hline${ }^{1} \mathrm{H}$ Literature & ${ }^{1} \mathbf{H}$ & ${ }^{13} \mathrm{C}$ Literature & ${ }^{13} \mathrm{C}$ \\
\hline $6.31, \mathrm{br}, 1 \mathrm{H}$ & $6.38, \mathrm{br}, 1 \mathrm{H}$ & 170.7 & 169.8 \\
\hline $5.88, \mathrm{br}, 1 \mathrm{H}$ & 5.91, br, 1H & 141.9 & 141.0 \\
\hline \multirow{2}{*}{$5.10, \mathrm{~m}, 3 \mathrm{H}$} & \multirow{2}{*}{ 5.12-5.07, m, 3H } & 135.3 & 135.6 \\
\hline & & 135.0 & 135.1 \\
\hline 4.11, d, $J=10.1 \mathrm{H}, 1 \mathrm{H}$ & 4.12, d, $J=11.0 \mathrm{~Hz}, 1 \mathrm{H}$ & 131.2 & 131.4 \\
\hline 4.06, ddd, $J=11.0,3.8,1.6 \mathrm{~Hz}, 1 \mathrm{H}$ & 4.06, ddd, $J=11.0,4.0,1.5 \mathrm{~Hz}, 1 \mathrm{H}$ & 125.8 & 127.1 \\
\hline $3.15, \mathrm{t}, J=11.0 \mathrm{H}, 1 \mathrm{H}$ & $3.17, \mathrm{t}, J=11.0 \mathrm{H}, 1 \mathrm{H}$ & 124.4 & 124.5 \\
\hline 2.06,m, 4H & & $124.2(2 \mathrm{C})$ & 124.3 and 124.2 \\
\hline \multirow{2}{*}{$2.00, \mathrm{~m}, 2 \mathrm{H}$} & & 76.0 & 76.2 \\
\hline & & 73.9 & 74.1 \\
\hline $1.99, \mathrm{~m}, 4 \mathrm{H}$ & \multirow[t]{3}{*}{ 2.11-1.91, m, 12H } & $39.7(2 \mathrm{C})$ & 39.9 and 39.8 \\
\hline $1.94, \mathrm{~m}, 1 \mathrm{H}$ & & 35.3 & 35.3 \\
\hline $1.91, \mathrm{~m}, 1 \mathrm{H}$ & & 32.5 & 32.6 \\
\hline \multirow{2}{*}{$1.68, \mathrm{~s}, 3 \mathrm{H}$} & \multirow{2}{*}{$1.68, \mathrm{~d}, J=1.0 \mathrm{~Hz}, 3 \mathrm{H}$} & 32.1 & 32.1 \\
\hline & & 30.4 & 30.4 \\
\hline $1.60, \mathrm{~m}, 1 \mathrm{H}$ & 1.64-1.57, m, 1H & 26.8 & 26.9 \\
\hline \multirow{2}{*}{$1.60, \mathrm{~s}, 9 \mathrm{H}$} & \multirow{2}{*}{$1.60, \mathrm{~s}, 9 \mathrm{H}$} & 26.6 & 26.7 \\
\hline & & 25.7 & 25.8 \\
\hline $1.30, \mathrm{~m}, 1 \mathrm{H}$ & \multirow[t]{2}{*}{ 1.38-1.30, m, 1H } & 25.0 & 25.0 \\
\hline 1.22, m, 1H & & 17.7 & 17.8 \\
\hline $1.15, \mathrm{~m}, 2 \mathrm{H}$ & 1.27-1.12, m, 3Н & $16.0(2 \mathrm{C})$ & $16.2(2 \mathrm{C})$ \\
\hline
\end{tabular}

